\title{
Special Issue on 2nd International Conference on Smart Systems Engineering (SmaSys 2014)
}

\author{
Ajit Khosla ${ }^{1} \cdot$ Hidemitsu Furukawa $^{1} \cdot$ Bernd Michel $^{2}$
}

Received: 16 September 2015 / Accepted: 29 September 2015 / Published online: 22 October 2015

(C) Springer-Verlag Berlin Heidelberg 2015

This special issue is devoted to Smart Systems. It has been an exciting opportunity to collect together papers from authors who were invited to present talks at the 2nd International Conference of Smart Systems Engineering 2014, October 15-17, 2014 Denkokuno-mori, Yonezawa, Japan. This conference attracted scientists and researchers from all over the world, who presented and discussed the challenges and emerging technologies in the smart materials, devices, and systems.

As we look forward, the pace of development in Smart Systems has accelerated, we anticipate that with the continued integration of new materials, sensors, and electronics, the widespread use of Smart Systems at economically viable costs will become a reality and will improve the wellbeing of our society in Japan and worldwide.

We would like to thank Prof. Hiroyuki Furusawa (SmaSys 2014, conference chair), all the organizing committee members, specially iFront staff and students for their effort to make this conference and special issue a success.

We would like to acknowledge the sponsors of the conference: Japan Society for the Promotion of Science (JSPS), Ministry of Education, Culture, Sports, Science and Technology, Japan (MEXT), The Society of Polymer
Science, Japan (SPSJ), Life-3D Printing Innovation Center (LPIC), Yamagata University, Soft and Wet Matter Engineering Laboratory (SWEL), Yamagata University, Yonezawa Alumni Association, Yamagata University, Yonezawa Convention Bureau, and Graduate School of Science and Engineering, Yamagata University.

We look forward to your participation in future special issues of Smart Systems.

Sincerely

Guest Editors

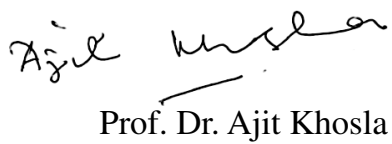

Hidentsu Furukawa

Prof. Dr. Hidemitsu Furukawa

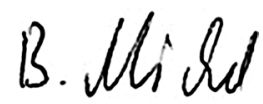

Prof. Bernd Michel,

Editor-in-Chief. Microsystem Technologies

Ajit Khosla

Khosla@gmail.com

Yamagata University, Yonezawa City, Yamagata, Japan

2 Micro Materials Center, Microsystem Technologies, Berlin, Germany 